\title{
Rising Tides on Black Holes
}

\author{
New calculations show that spinning black holes-unlike nonspinning \\ ones-can be tidally deformed by a nonsymmetric gravitational field.
}

By Michael Schirber

C ould a black hole have tides? The common view has been no, these dense objects are too rigid to form such surface deformations. But a new theoretical calculation finds that a spinning black hole can develop a tidal bulge in a nonaxisymmetric gravitational field [1]. Alexandre Le Tiec from the Observatory of Paris and Marc Casals from the Brazilian Center for Research in Physics show that these conditions could arise during a black hole merger and that the bulging should affect the black hole's spin.

Earth changes its shape under the pull of the Moon and the Sun. This deformability is characterized by the "tidal Love numbers" (TLNs), named after the mathematician Augustus Love. Various TLNs describe different modes of tidal response. Earth's quadrupolar TLN, for example, has a value of 0.3 , while the same parameter for a (less deformable) neutron star is estimated to be around 0.1 .

Previous work established that the TLNs are zero for a nonspinning black hole in a static tidal field. But the situation is different when the field changes with time or when the black hole spins in an asymmetric field. Le Tiec and Casals found that

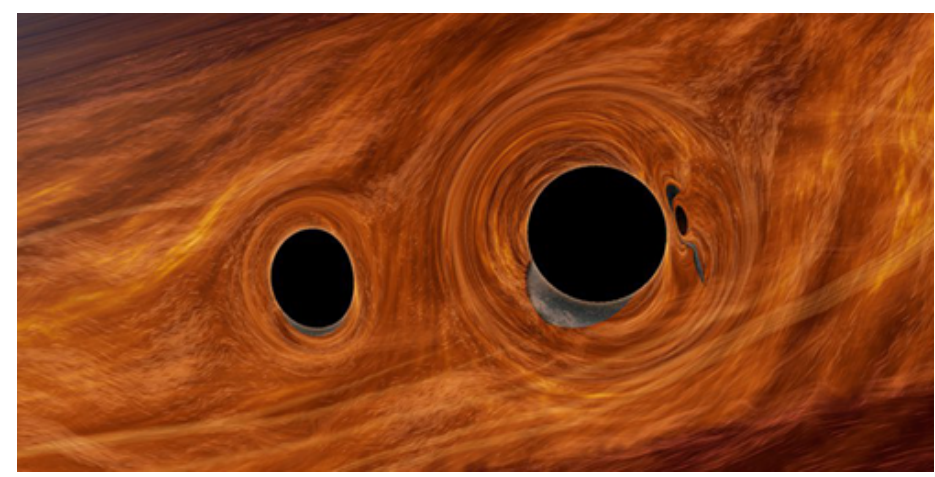

Credit: Caltech/R. Hurt (IPAC) a black hole spinning at $10 \%$ of the maximum rate in an asymmetric field should have a quadrupolar TLN of 0.002 . Despite being relatively small, such tidal deformations could influence the dynamics of black hole mergers. For the case of a stellar-mass black hole spiraling into a spinning supermassive black hole, they found that tidal bulges on the larger object generate a torque that slows the spinning down.

Michael Schirber is a Corresponding Editor for Physics based in Lyon, France.

\section{REFERENCES}

1. A. Le Tiec and M. Casals, "Spinning black holes fall in Love," Phys. Rev. Lett. 126, 131102 (2021). 